\title{
Assessing Facial Skin Temperature Asymmetry with Different Methods
}

\author{
by N. Ludwig*, D. Formenti ${ }^{* *}$ A. Rossi ${ }^{* *}$, A. Trecroci* ${ }^{* *}$ M. Gargano* and G. Alberti \\ *Department of Physics, Università degli Studi di Milano, Italy. nicola.ludwig@unimi.it \\ **School of Exercise Sciences, Department of Biomedical Sciences for Health, Università degli Studi di Milano, \\ Italy. damiano.formenti@unimi.it
}

\section{Abstract}

The analysis of thermographic images in psychological application is usually performed by averaging the values of pixel temperature inside a Region of Interest (Troi). We used a new method, based on maximal temperatures inside a $\mathrm{ROI}$ (Tmax), to evaluate face temperature to single out asymmetry in males and females population ( $\mathrm{n}=33$ and 27 respectively). Data were compared with those obtained with traditional method based on the average over the whole area of the anatomical part under investigation (hemiface). In this study, we put in evidence the features of this analysis method specifically in human non-invasive facial characterization.

\section{Introduction}

Lately, in the psychophysiological sciences Infrared Thermography (IRT) progressively turned into a widespread technique to detect variations in skin temperature depending on emotional conditions. Several authors measured skin temperatures of different zones of the face during different exciting conditions [1-3].

The thermal images analysis is commonly performed by a dedicated software that allows to select a Region of Interest $(\mathrm{ROI})$ on the body surface and to calculate the mean value and its standard deviation over all the pixels inside such ROI. Even if the most common method used in skin temperature evaluation is Troi, we applied an alternative method based on maximal temperatures detection already tested in several trials to evaluate the skin temperature during physical exercise. Main work has been done on calves during and after exercise [4] in a young female population, and on thighs during squat exercise in an active male population [5]. The systematic comparison between the different methods was studied in rest condition on calves of females subjects [6]. We found that despite higher value of Tmax, the two methods have good agreement.

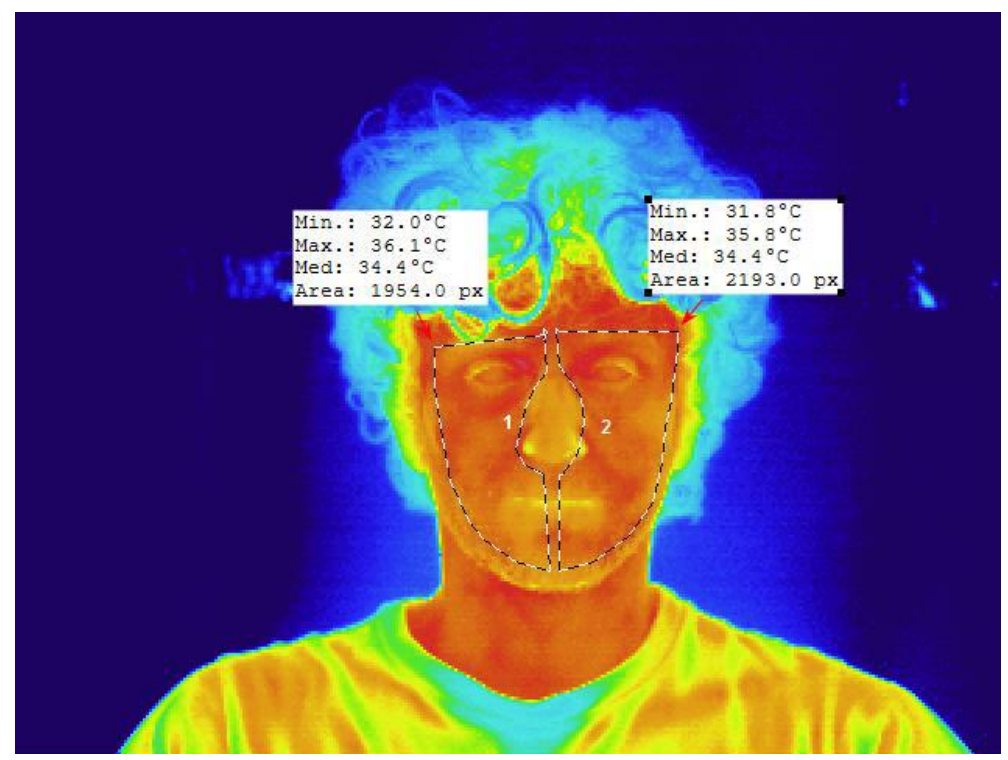

Fig. 1. Region of interest in the two hemifaces in the thermal image of a representative subject, software gives mean, maximum, minimum temperature and area in each of the two hemifaces; standard deviation was calculated separately.

In this study we aimed to compare the Troi and Tmax methods [6] in the evaluation of facial skin temperature asymmetry in males and females healthy subjects. Thermal images of the face of 60 subjects in resting condition were recorded and subsequently analysed using Troi and Tmax methods. We hypothesized that differences between Troi and Tmax methods would be found to detect facial asymmetry. In oreder to emphasise the homogeneity of the sample, only 


\subsection{1/qirt.2016.074}

students of the same faculty and age was taken into account. As a first attempt to find out any possible asymmetry, only the gender has been considered as a discriminating factor.

\section{Methods}

Sixty young active healthy subjects, (males $n=33$ and females $n=27$ ) took part voluntarily in this study. They belonged to the student population of the School of Exercise Sciences of the Università degli Studi di Milano. Their mean age and body mass index were $21.1 \pm 2.1$ years, $21.8 \pm 1.9 \mathrm{~kg} / \mathrm{m}^{2}$, respectively. All participants were non smokers, and without cardiovascular or pulmonary diseases. They declared to have not assumed drugs or medications with a potential effect on cardiovascular and thermoregulatory functions during the two months prior the tests. The Ethical Committee of the State University of Milan approved this study.

All the thermal images were recorded in laboratory conditions, with environmental temperature and relative humidity controlled $\left(22-23^{\circ} \mathrm{C}, 50 \pm 5 \%\right)$. Three thermal images were recorded for each subject in resting condition after a room acclimation period of 15 minutes. Temperature were analyzed using a dedicated software for extracting temperature values with Troi and Tmax methods. For the first method an operator selected a ROI per each hemiface avoiding nose region as showed in Figure 1.

The values of Tmax method was obtained using the temperature of the five hottest pixels for each of the four anatomical regions under consideration: lips ( $\mathrm{La})$, cheek (Ca), orbital (Oa) and supraorbital (SOa) as shown in Figure 2.

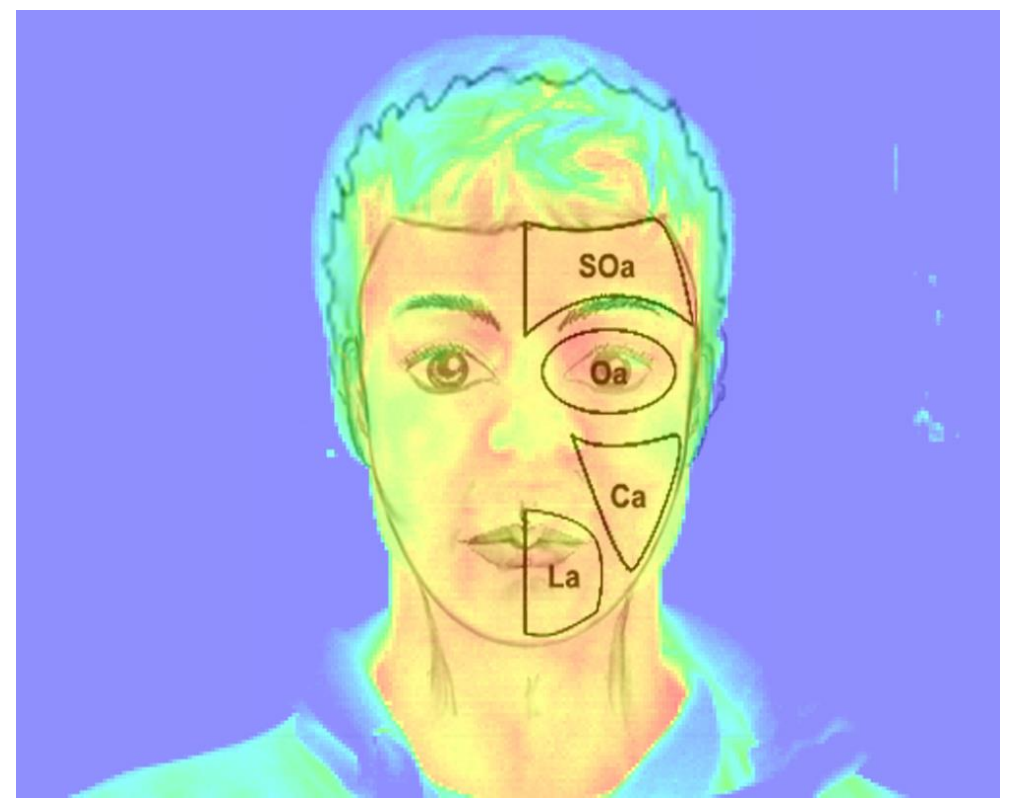

Fig. 2. Anatomical regions for temperature analysis with Tmax method in right hemiface.

These pixels were selected imposing a minimum distance of 5 pixels among each other. Finally, its temperature value was obtained averaging over an area of $5 \times 5$ pixels around the hottest five ones. In this way the result represented an overall amount of 500 pixels per hemiface. In this way, for every thermal image, each subject was represented by a temperature value calculated using the same number of pixels, which were representative of cutaneous area essential for heat dissipation. We stress that the Troi method, on the other side, considered areas ranging between 2000 and 3000 pixels. Such procedure derived from the one previously used for calf temperature [6], whereas in this study it was used to highlight differences related to different anatomical regions inside the face.

Paired t-test was used to investigate the differences in skin temperature between right and left hemifaces in each method, thus identifying which method was the most suitable for asymmetry detection, as well as to detect differences between Troi and Tmax. Pearson correlation coefficient was calculated between left and right values in each method in overall sample, and in male and female population.

Due to the huge amount of data and the need of an accurate manual selection for the Troi, this method was applied in this preliminary phase only for $10 \mathrm{M}+10 \mathrm{~F}$ subjects.

\section{Results}

Figures 3 and 4 show the relationship between left and right hemifaces in males and females calculated by Tmax method (60 subjects) as well as Troi ( 20 subjects). In order to take into account temperatures coming from all the different parts of the face, Tmax was obtained at a first step separately in the four areas of figure 2 and further averaged to give a single value representative of that method for the whole hemiface. 


\subsection{1/qirt.2016.074}

\subsection{Tmax method results}

Using Tmax method (Figure 3) with the procedure of averaging over 4 areas of each hemiface as aforesaid we found a difference between left and right hemifaces only in female population $(n=27, p>0.05)$. The angular coefficient of the linear regression best fit of left vs. right temperature values for female subjects is in fact 0.88 whereas for male subjects we found 0.98 . This last result can be considered as an indication of high symmetry between right and left temperature hemifaces. In both series of data correlation index is extremely high $\left(R^{2}>0.90\right)$.

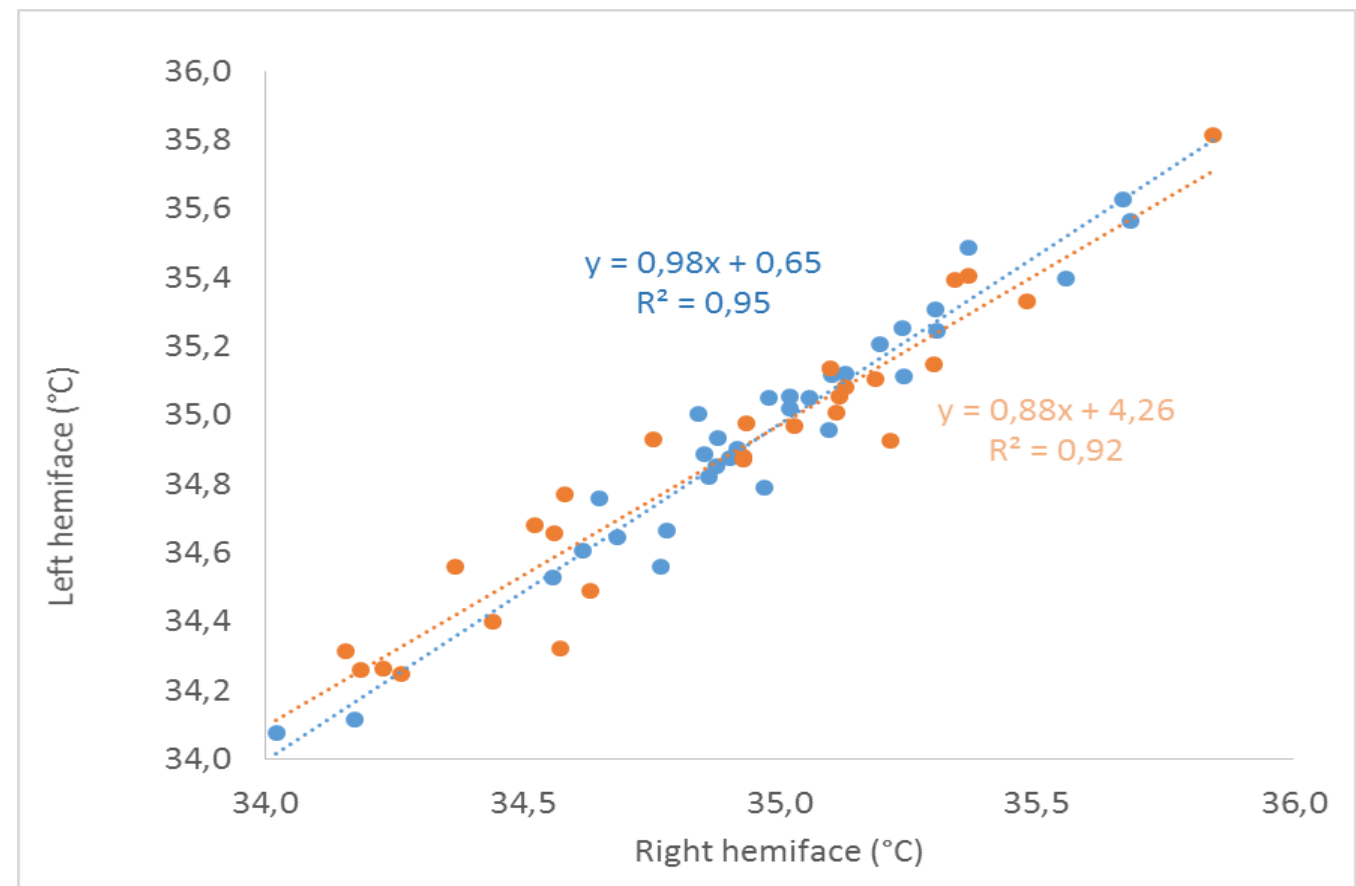

Fig. 3. Temperature of left and right hemiface calculated by Tmax. Male data are in blue, female in orange.

In Table 1 this kind of analyses was done on the four different areas of each hemifaces considered (i.e. Soa, $\mathrm{Oa}, \mathrm{Ca}$, La as indicated in fig.2). This detailed analisys allowed us first to confirm that female subjects are the most asymmetric one and, second that the areas where asymmetry occurs are Ca and La (cheeks and lips). Finally the eye region $(\mathrm{Oa})$ seems to be the one where temperatures spread more between left and right side of the face.

Tab. 1. Angular coefficients of the linear regression best fit of left vs. right areas on the hemifaces. Temperature was obtained using Tmax method on different areas of the hemiface (supraorbital, orbital, cheek, lips), in bold the lower values, considered those farther from simmetry.

\begin{tabular}{lcccc}
\hline $\begin{array}{l}\text { ANGULAR } \\
\text { COEFFICIENT }\end{array}$ & $\begin{array}{c}\text { Supraorbital } \\
(\text { Soa })\end{array}$ & $\begin{array}{c}\text { Orbital } \\
(\mathrm{Oa})\end{array}$ & $\begin{array}{c}\text { Cheek } \\
(\mathrm{Ca})\end{array}$ & $\begin{array}{c}\text { Lips } \\
(\mathrm{La})\end{array}$ \\
\hline Total $(\mathrm{n}=60)$ & $0.98\left(\mathrm{R}^{2}=.92\right)$ & $\mathbf{0 . 7 5}\left(\mathrm{R}^{2}=.73\right)$ & $0.89\left(\mathrm{R}^{2}=.91\right)$ & $0.92\left(\mathrm{R}^{2}=.89\right)$ \\
Male $(\mathrm{n}=33)$ & $0.99\left(\mathrm{R}^{2}=.92\right)$ & $\mathbf{0 . 8 0}\left(\mathrm{R}^{2}=.73\right)$ & $1.00\left(\mathrm{R}^{2}=.92\right)$ & $0.98\left(\mathrm{R}^{2}=.93\right)$ \\
Female $(\mathrm{n}=27)$ & $0.98\left(\mathrm{R}^{2}=.91\right)$ & $\mathbf{0 . 7 3}\left(\mathrm{R}^{2}=.74\right)$ & $\mathbf{0 . 8 4}\left(\mathrm{R}^{2}=.90\right)$ & $\mathbf{0 . 8 4}\left(\mathrm{R}^{2}=.84\right)$ \\
\hline
\end{tabular}




\subsection{1/qirt.2016.074}

\subsection{Troi method results}

Using Troi method the temperature differences (asymmetry) found in females subjects $(n=10, p>0.05$, in orange Fig. 4) were higher than those obtained with Tmax method. Angular coefficient value for female is in fact $0.82\left(R^{2}=0.95\right)$ whereas in male population (Fig. 4 in blue) it is $1.05\left(n=10, R^{2}=0.99, p>0.05\right)$.

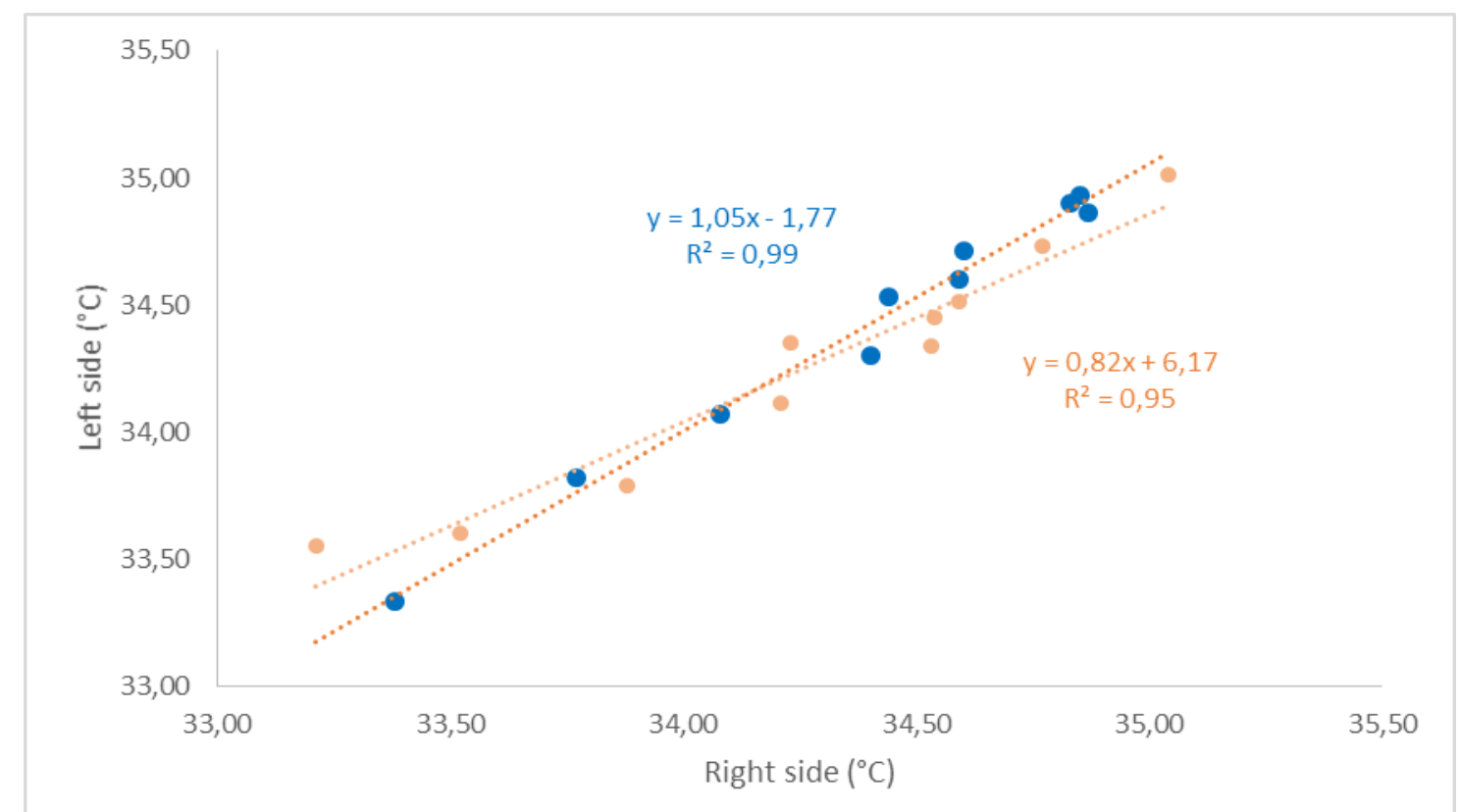

Fig. 4. Relationship between left and right hemifaces calculated by Troi. The data of male subjects are in blue, female in orange.

Finally, we compared the data with the Tmax method. Figure 5 shows the correlation between all the data (M and $F$, left and right) obtained through the two procedures of analysis $(n=40, p<0.0001)$. Angular coefficient $(0.81)$ far from the unity represents the difference due to pixel selection method and can be assumed without diagnostic meaning. High value of Pearson's coefficient $\left(R^{2}=0.986\right)$ is comparable to the ones obtained in other research studies $[4,6]$ on leg muscles.

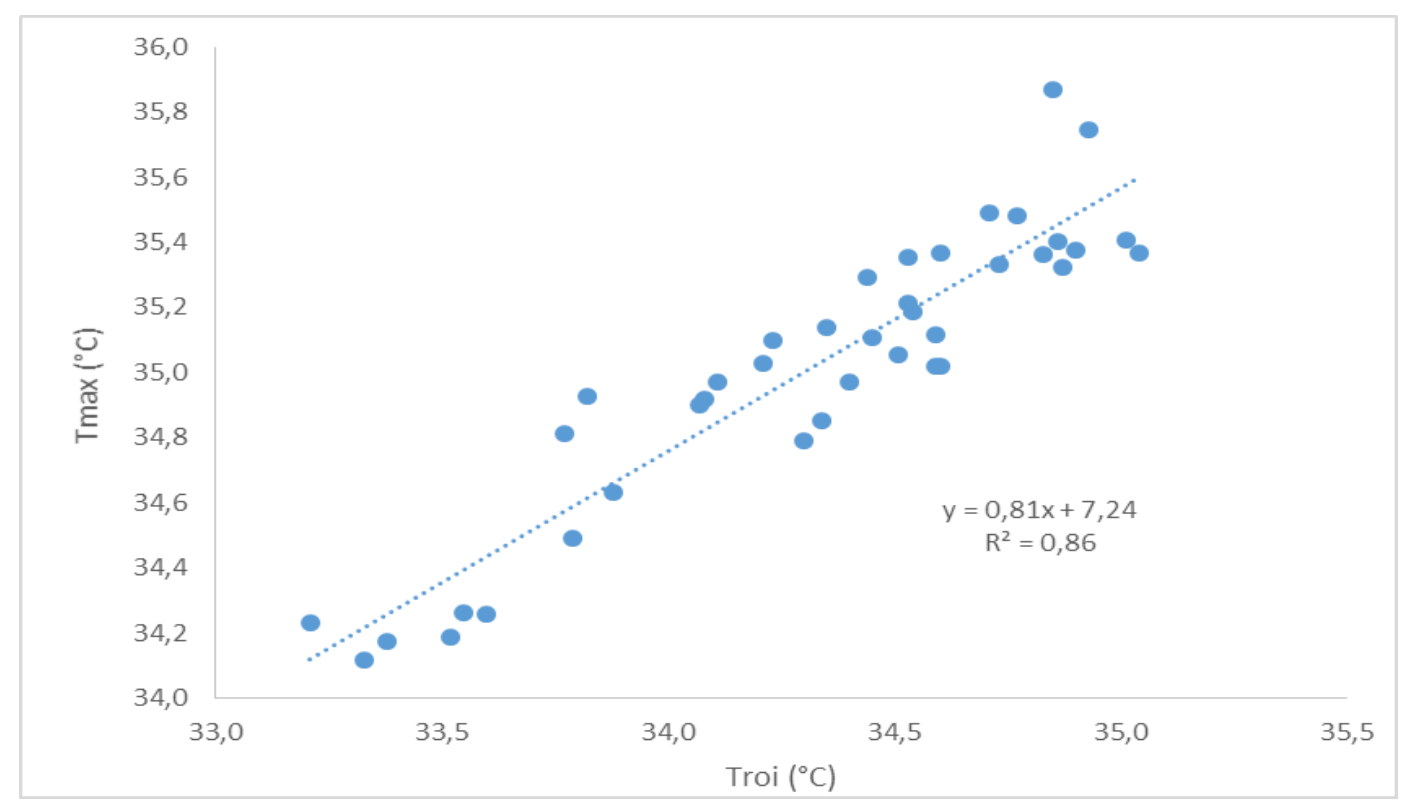

Figure 5 Comparison between Tmax and Troi values obtained from the 40 hemifaces. 


\subsection{1/qirt.2016.074}

The comparison of the two methods to single out differences in temperature between right and left hemifaces of the two groups of subjects allowed us further considerations. In particular in this preliminary research study it seems that Troi method is more able to highlight differences in temperature between the two hemifaces at rest condition with respect to the Tmax method. This is probably due to the fact that maximal values of temperature of people tend (in nonpathological condition) to the average value influenced by the inner metabolism which is not affected by cutaneous specific (depending on subjects) conditions.

\subsection{Errors evaluation}

For clarity, error bars, as standard deviations from the mean value, were not drawn into the graphs. However, as a final remark we stress that in such a variable different ROI from the physiological point of view for Troi their values range $0.5-0.9^{\circ} \mathrm{C}$, with a mean value of $0.67^{\circ} \mathrm{C}$. Otherwise, Tmax values show to stay below $0.2^{\circ} \mathrm{C}$, sure due to a higher homogeneity of the pixels chosen.

A final analysis of the maxima method was done in order to evaluate any possible dependence of temperature measured from the traditional method of averaging temperature over an region of interest. Figure 6 show the Bland and Altman plot of Troi and Tmax methods, considering Troi as golden standard. It demonstrates that error are without any temperature dependence and single out the systematic difference of $+0.7^{\circ} \mathrm{C}$ between the two systems of analysis.

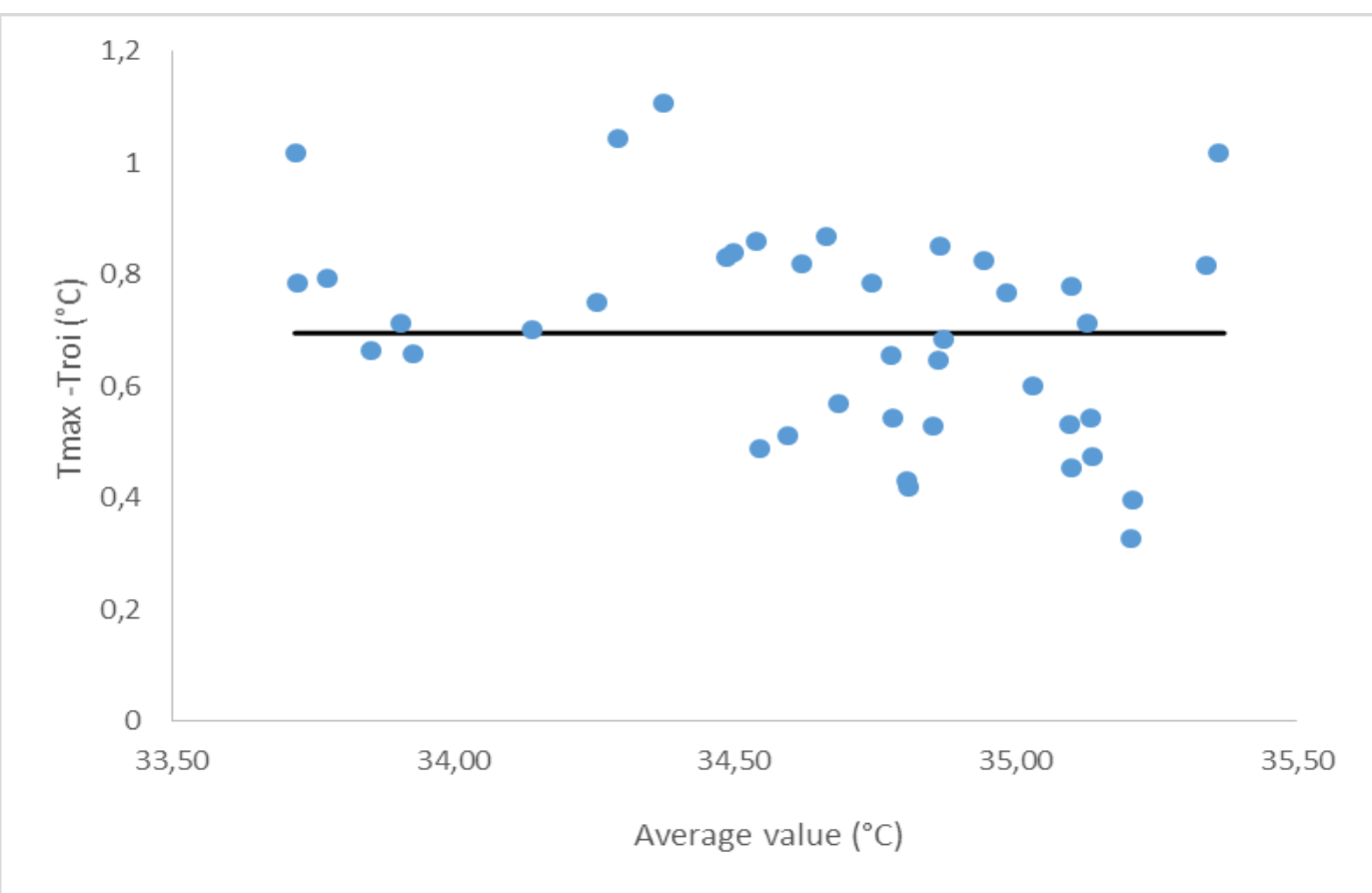

Figure 6 Bland and Altman test to verifay dispersion of Tmax data with respect to Troi considered as golden standard. Comparison was made taking into account only the 20 subjects used in Troi analysis.

\section{Conclusion}

Our data showed that Troi was the most suitable method to highlight possible skin temperature asymmetry between left and right side of the face. In particular, a difference between left and right side of face seems to take place has been observed only for female group.

Therefore, our results support the use of thermography in psychophysiological studies aimed to investigate thermal asymmetry [7] and suggest the use of Troi as the most useful method of analysis. The significant difference between Troi and Tmax method is due to the peculiarity of Tmax method in considering pixels only with higher temperature values [6]. Despite this systematic difference, we stress that Tmax method demonstrated a higher precision in temperature evaluation with respect to Troi. This is due to the small amount of pixels involved in the calculation of the mean itself.

We believe that the present research contributes to characterize both Troi and Tmax methods to calculate skin temperature on facial asymmetry at rest by using infrared thermography. The skin temperature obtained by both method showed a good agreement. 


\section{REFERENCES}

[1] Ioannou, S., Gallese, V., Merla, A., Thermal infrared imaging in psychophysiology: potentialities and limits. Psychophysiology 2014, 51, 951-963.

[2] Ring E.F.J., Ammer K., Infrared thermal imaging in medicine, Physiol Meas. 33 (2012) R33-46.

[3] Lahiri B.B., Bagavathiappan S., Jayakumar T., Philip J., Medical applications of infrared thermography: A review, Infrared Physics \& Technology. 55 (2012) 221-235.

[4] Formenti, D., Ludwig, N., Gargano, M., Gondola, M., et al., Thermal Imaging of Exercise-Associated Skin Temperature Changes in Trained and Untrained Female Subjects. Ann. Biomed. Eng. 2013, 41, 863-871.

[5] Formenti, D., Ludwig, N., Trecroci, A., Gargano, M., et al., Dynamics of thermographic skin temperature response during squat exercise at two different speeds. J. Therm. Biol. Accepted and in press

[6] Ludwig, N., Formenti, D., Gargano, M., Alberti, G., Skin temperature evaluation by Infrared Thermography: Comparison of Image Analysis Methods. Infrared Phys. Technol. 2014, 62, 1-6.

[7] Wright C.I., Kroner C.I., Draijer R., Non-invasive methods and stimuli for evaluating the skin's microcirculation, $J$ Pharmacol Toxicol Methods. 54 (2006) 1-25. 